УДК 539.374

\title{
Antiplane Axisymmetric Creep Deformation of Incompressible Medium
}

\author{
Sergey N. Firsov * \\ Aleksandr N. Prokudin ${ }^{\dagger}$ \\ Institute of Machinery and Metallurgy FEB RAS \\ Metallurgov, 1, Komsomolsk-on-Amur, 681005 \\ Russia
}

Received 10.05.2015, received in revised form 24.06.2015, accepted 11.09.2015

Flow of incompressible medium under varying gradient of pressure is considered. It is assumed that medium exhibits nonlinear elastic and creep behavior. The theory of large strains based on transport equations for the tensors of reversible and irreversible deformations is used for problem formulation. Analytical and numerical methods are applied to solve the problem.

Keywords: large strain, elasticity, creep, springback.

DOI: 10.17516/1997-1397-2015-8-4-406-415

\section{Introduction}

Model problems with simplified geometry and kinematics has a great value for developing theories of mechanical behavior of materials. Analytical solutions of such problems allow one to perform qualitative analysis of investigated process and to verify numerical solutions. Anti-plane deformation problem is one of the simplest model problems. This problem was solved for linear elastic medium, nonlinear elastic medium and elastoplastic medium. One should mention the set of papers [1-5] published by V.D. Bondar in which anti-plane deformation problem is solved in the frameworks of finite strain elasticity and elastoplasticity.

In this paper anti-plane deformation problem is solved in the context of theory of large elastoplastic deformations. This theory is based on non-equilibrium thermodynamics formalism. It assumes that irreversible and reversible deformations are defined by differential transport equations.

We consider flow of incompressible medium within cylindrical tube due to pressure gradient. No-slip boundary condition is set on the walls of the tube. The points of the medium are restricted to move only along lines parallel to the element of the cylinder. We suppose that medium deforms both reversibly and irreversibly. In addition, irreversible deformation accumulation is due to creeping of medium. Present work is the continuation of the research [6,7] in which similar problem statement was used. The main distinction of works [6,7] is that irreversible deformation of medium is due to plastic flow.

\footnotetext{
*firsov.s.new@yandex.ru

†p prokudin@imim.ru

(C) Siberian Federal University. All rights reserved
} 


\section{Mathematical model}

Kinematics of continuum is described by spatial (Euler) coordinates. For simplicity all equations are given in Cartesian coordinate system. We use the Almansi finite strain tensor:

$$
d_{i j}=\frac{1}{2}\left(u_{i, j}+u_{j, i}-u_{k, i} u_{k, j}\right) .
$$

Here $u_{i}$ are the components of displacement vector.

Let us consider reversible and irreversible strains along with temperature and entropy as state variables. We denote the components of reversible and irreversible strains as $e_{i j}$ and $p_{i j}$, respectively. In accordance with $[8,9]$ differential transport equations are written as

$$
\begin{gathered}
\frac{D e_{i j}}{D t}=\varepsilon_{i j}-\gamma_{i j}-\frac{1}{2}\left(\left(\varepsilon_{i k}-\gamma_{i k}+z_{i k}\right) e_{k j}-e_{i k}\left(\varepsilon_{k j}-\gamma_{k j}+z_{k j}\right)\right), \\
\frac{D p_{i j}}{D t}=\gamma_{i j}-p_{i k} \gamma_{k j}-\gamma_{i k} p_{k j}
\end{gathered}
$$

where

$$
\begin{gathered}
\frac{D n_{i j}}{D t}=\frac{d n_{i j}}{d t}-r_{i k} n_{k j}+n_{i k} r_{k j}, \quad \frac{d n_{i j}}{d t}=\frac{\partial n_{i j}}{\partial t}+n_{i j, j} v_{j}, \\
r_{i j}=w_{i j}+z_{i j}\left(e_{i j}, \varepsilon_{i j}\right), \quad w_{i j}=\frac{1}{2}\left(v_{i, j}-v_{j, i}\right), \quad \varepsilon_{i j}=\frac{1}{2}\left(v_{i, j}+v_{j, i}\right), \\
z_{i j}=-z_{j i}=A^{-1}\left(B^{2}\left(\varepsilon_{i k} e_{k j}-e_{i k} \varepsilon_{k j}\right)+B\left(\varepsilon_{i k} e_{k m} e_{m j}-e_{i k} e_{k m} \varepsilon_{m j}\right)+\right. \\
\left.+e_{i k} \varepsilon_{k m} e_{m n} e_{n j}-e_{i k} e_{k m} \varepsilon_{m n} e_{n j}\right), \\
A=8-8 E_{1}+3 E_{1}^{2}-E_{2}-\frac{1}{3} E_{1}^{3}+\frac{1}{3} E_{3}, \quad B=2-E_{1}, \\
E_{1}=e_{j j}, \quad E_{2}=e_{i j} e_{j i}, \quad E_{3}=e_{i j} e_{j k} e_{k i} .
\end{gathered}
$$

Here $\frac{D}{D t}$ is the objective derivative operator, $\frac{d}{d t}$ is the total derivative operator, $\frac{\partial}{\partial t}$ is the partial derivative operator, $r_{i j}$ are the components of rotation tensor, $w_{i j}$ are the components of the vorticity tensor, $\varepsilon_{i j}$ are the components of strain rate tensor, $v_{i}$ are the components of velocity vector, $z_{i j}$ is nonlinear term of the vorticity tensor, $E_{i}$ are the invariants of reversible deformations tensor.

If we assume that there is no nonlinear term in the vorticity tensor, then objective derivative $\frac{D n_{i j}}{D t}$ introduced above coincides with the Jaumann derivative.

The separation of the total strain into reversible and irreversible strains follows from (2) and has the form

$$
d_{i j}=e_{i j}+p_{i j}-\frac{1}{2} e_{i k} e_{k j}-e_{i k} p_{k j}-p_{i k} e_{k j}+e_{i k} p_{k m} e_{m j}
$$

Stresses in the medium are determined by relations that are similar to the Murnaghan relations known from the theory of nonlinear elasticity:

$$
\sigma_{i j}=-P_{1} \delta_{i j}+\frac{\partial W}{\partial e_{i k}}\left(\delta_{k j}-e_{k j}\right), p_{i j} \neq 0 .
$$

Here $\sigma_{i j}$ are the components of the Euler-Cauchy stress tensor, $P_{1}$ is the additional hydrostatic pressure and $W$ is the elastic potential. 
When there are no irreversible strains in the medium $\left(p_{i j} \equiv 0\right)$ relation $(5)$ coincides with the Murnaghan relations.

We take the Helmholtz free energy as the thermodynamic potential in the relation (5). If we assume that the free energy distribution density does not depend on irreversible deformations then $W=\rho_{0} \psi$. In this case elastic potential for isotropic incompressible medium can be written as Taylor series expansion in respect to free state. For simplicity we keep terms up to the third order:

$$
\begin{gathered}
W=W\left(J_{1}, J_{2}\right)=(\alpha-\mu) J_{1}+\alpha J_{2}+\beta J_{1}^{2}-\xi J_{1} J_{2}-\zeta J_{1}^{3}, \\
J_{1}=s_{j j}, \quad J_{2}=s_{i j} s_{j i}, \quad s_{i j}=e_{i j}-\frac{1}{2} e_{i k} e_{k j} .
\end{gathered}
$$

Here $\mu, \alpha, \beta, \xi, \zeta$ are elastic moduli and $\mu$ is the share modulus.

Upon substituting elastic potential expansion into (5), we obtain relations between stresses and reversible deformations:

$$
\begin{aligned}
\sigma_{i j}=a_{0} \delta_{i j}+ & 2 a_{1} e_{i j}+a_{2} e_{i k} e_{k j}-4 a_{3} e_{i s} e_{s t} e_{t j}+a_{3} e_{i s} e_{s t} e_{t k} e_{k j}, \\
a_{0} & =-P_{1}-2 \mu+2 b I_{1}-(b-\mu) I_{2}-3 \zeta I_{1}^{2}, \\
a_{1} & =\mu+(b+\mu) I_{1}+(\mu-b) I_{2}+3 \zeta I_{1}^{2}, \\
a_{2} & =3 \mu+(5 \mu-3 b) I_{1}+(b-\mu) I_{2}-3 \zeta I_{1}^{2}, \\
a_{3} & =\mu+(\mu-b) I_{1} .
\end{aligned}
$$

Let us assume that irreversible deformation accumulation is due to creeping of medium and it occurs during the whole process.

The source of irreversible strains $\gamma_{i j}$ in transport equations (2) has the form

$$
\gamma_{i j}=\varepsilon_{i j}^{v}=\frac{\partial V(\Sigma)}{\partial \sigma_{i j}}
$$

Let us write the dissipative potential in (8) according to the Norton creep power law [10]:

$$
V(\Sigma)=B \Sigma^{n}\left(\sigma_{1}, \sigma_{2}, \sigma_{3}\right), \quad \Sigma=\sqrt{\frac{3}{2}\left(\left(\sigma_{1}-\sigma\right)^{2}+\left(\sigma_{2}-\sigma\right)^{2}+\left(\sigma_{3}-\sigma\right)^{2}\right)}, \quad \sigma=\frac{1}{3} \sigma_{k k} .
$$

Here $\sigma_{i}$ are the principal values of the Euler-Cauchy stress tensor and $B, n$ are the creep parameters of medium which can be determined experimentally.

One should note that any creep law which is suitable for specific problem can be used in the relation (8) instead of the Norton law.

\section{Problem statement}

For convenience we use cylindrical coordinate system $r, \varphi, z$. Assume that an incompressible medium fills a cylindrical tube of radius $R$. Deformation of medium is due to time-dependent pressure gradient:

$$
\frac{\partial P_{1}}{\partial z}=-\psi(t)
$$

Initially the medium is undeformed and pressure gradient is equal to zero.

No-slip conditions are set on the tube walls:

$$
\left.\vec{u}\right|_{r=R}=\left.\vec{v}\right|_{r=R}=\overrightarrow{0} .
$$


Let us assume that the unknown displacement and velocity vectors have one nonzero component $\left(u_{z}\right.$ and $v_{z}$, respectively). Taking into account axial symmetry, we have

$$
u=u_{z}(r, t), \quad v=v_{z}(r, t) .
$$

In this case nonzero components of Almansi strain tensor are

$$
d_{r r}=-\frac{1}{2}\left(\frac{\partial u}{\partial r}\right)^{2}, \quad d_{r z}=d_{z r}=\frac{1}{2} \frac{\partial u}{\partial r} .
$$

According to assumed continuum kinematics, we have following relations for nonzero components of strain rate tensor, vorticity tensor and rotation tensor

$$
\varepsilon_{r z}=\varepsilon_{z r}=\frac{1}{2} \frac{\partial v}{\partial r} ; \quad w_{z r}=-w_{r z}=\frac{1}{2} \frac{\partial v}{\partial r} ; \quad r_{r z}=-r_{z r}=\frac{2 \varepsilon_{r z}\left(1-e_{z z}\right)}{e_{r r}+e_{z z}-2} .
$$

In accordance with constraints (12) the operator of total derivative in cylindrical coordinate system has the form

$$
\frac{d n_{i j}}{d t}=\frac{\partial n_{i j}}{\partial t}+v_{z} \frac{\partial n_{i j}}{\partial z} .
$$

The introduced kinematic constraint on medium motion imposes limitations on the form of elastic potential (6) [11] . It turns out that some coefficients in Taylor expansion (6) are not independent and, taking into account (12), the elastic potential has the form

$$
W=W\left(J_{1}, J_{2}\right)=-2 \mu J_{1}-\mu J_{2}+b J_{1}^{2}+(b-\mu) J_{1} J_{2}-\zeta J_{1}^{3} .
$$

Let us substitute relation (16) into (5). Then we take into account only first order terms in diagonal components of reversible deformation tensor and first and second order terms in non-diagonal components of irreversible deformation tensor. As a result of transformations we obtain the following relations for stresses in the medium:

$$
\begin{gathered}
\sigma_{r r}=-\left(P_{1}+2 \mu\right)+2 b\left(e_{r r}+e_{z z}+e_{\varphi \varphi}\right)+2 \mu e_{r r}+\mu e_{r z}^{2}, \\
\sigma_{\varphi \varphi}=-\left(P_{1}+2 \mu\right)+2 b\left(e_{r r}+e_{z z}+e_{\varphi \varphi}\right)+2 \mu e_{\varphi \varphi}-2 \mu e_{r z}^{2}, \\
\sigma_{z z}=-\left(P_{1}+2 \mu\right)+2 b\left(e_{r r}+e_{z z}+e_{\varphi \varphi}\right)+2 \mu e_{z z}+\mu e_{r z}^{2}, \\
\sigma_{r z}=2 \mu e_{r z} .
\end{gathered}
$$

Equilibrium equations in cylindrical coordinate system with axial symmetry without body forces have the form

$$
\begin{gathered}
\frac{\partial \sigma_{r r}}{\partial r}+\frac{\partial \sigma_{r z}}{\partial z}+\frac{\sigma_{r r}-\sigma_{\varphi \varphi}}{r}=0, \\
\frac{\partial \sigma_{r z}}{\partial r}+\frac{\partial \sigma_{z z}}{\partial z}+\frac{\sigma_{r z}}{r}=0 .
\end{gathered}
$$

Taking into consideration relations $(12-15)$, we write transport equations for components of irreversible deformations tensor. We also assume that components of reversible and irreversible deformations are independent of axial coordinate and thus their total derivative is the partial derivative:

$$
\begin{gathered}
\gamma_{r r}\left(1-2 p_{r r}\right)=\frac{\partial p_{r r}}{\partial t}+2 p_{r z}\left(\gamma_{r z}+r_{z r}\right) \\
\gamma_{z z}\left(1-2 p_{z z}\right)=\frac{\partial p_{z z}}{\partial t}+2 p_{r z}\left(\gamma_{r z}+r_{r z}\right) \\
\gamma_{\varphi \varphi}\left(1-2 p_{\varphi \varphi}\right)=\frac{\partial p_{\varphi \varphi}}{\partial t} \\
\gamma_{r z}\left(1-p_{r r}-p_{z z}\right)=\frac{\partial p_{r z}}{\partial t}+r_{r z}\left(p_{r r}-p_{z z}\right)+p_{r z}\left(\gamma_{z z}+\gamma_{r r}\right) .
\end{gathered}
$$


Transport equations for components of reversible deformations tensor take the form

$$
\begin{gathered}
-\gamma_{r r}=\frac{\partial e_{r r}}{\partial t}+2 r_{z r} e_{r z} \\
-\gamma_{z z}=\frac{\partial e_{z z}}{\partial t}+2 r_{r z} e_{r z} \\
-\gamma_{\varphi \varphi}=\frac{\partial e_{\varphi \varphi}}{\partial t} \\
-\frac{1}{2} \gamma_{r z}\left(2+e_{r r}-e_{z z}\right)=\frac{\partial p_{r z}}{\partial t}+r_{r z}\left(e_{r r}-e_{z z}\right)-\frac{1}{2} \varepsilon_{r z}\left(2+e_{r r}-e_{z z}\right)+e_{r z}\left(\gamma_{z z}-\gamma_{r r}\right)
\end{gathered}
$$

Source of irreversible deformations in the transport equations is

$$
\begin{gathered}
\gamma_{r r}=B n \Sigma^{n-2}\left(2 \sigma_{r r}-\sigma_{\varphi \varphi}-\sigma_{z z}\right) \\
\gamma_{z z}=B n \Sigma^{n-2}\left(2 \sigma_{z z}-\sigma_{\varphi \varphi}-\sigma_{r r}\right), \\
\gamma_{\varphi \varphi}=B n \Sigma^{n-2}\left(2 \sigma_{\varphi \varphi}-\sigma_{r r}-\sigma_{z z}\right), \\
\gamma_{r z}=6 B n \Sigma^{n-2} \sigma_{r z} \\
\Sigma=\sqrt{\left(\sigma_{r r}-\sigma_{\varphi \varphi}\right)^{2}+\left(\sigma_{r r}-\sigma_{z z}\right)^{2}+\left(\sigma_{z z}-\sigma_{\varphi \varphi}\right)^{2}+6 \sigma_{r z}^{2}} .
\end{gathered}
$$

Combining the above relation with equations (17), we can express rates of irreversible deformations in terms of reversible deformations:

$$
\begin{gathered}
\gamma_{r r}=B n \mu \Sigma^{n-2}\left(4 e_{r r}-2 e_{\varphi \varphi}-2 e_{z z}+3 e_{r z}^{2}\right) \\
\gamma_{z z}=B n \mu \Sigma^{n-2}\left(4 e_{z z}-2 e_{\varphi \varphi}-2 e_{r r}+3 e_{r z}^{2}\right) \\
\gamma_{\varphi \varphi}=B n \mu \Sigma^{n-2}\left(4 e_{\varphi \varphi}-2 e_{r r}-2 e_{z z}-6 e_{r z}^{2}\right) \\
\gamma_{r z}=12 B n \mu \Sigma^{n-2} e_{r z} .
\end{gathered}
$$

\section{Solution}

Stresses and strains in the medium are defined by the following unknown functions: $u(r, t)$, $v(r, t), P_{1}(r, z, t), e_{r r}(r, t), e_{z z}(r, t), e_{\varphi \varphi}(r, t), e_{r z}(r, t), p_{r r}(r, t), p_{z z}(r, t), p_{\varphi \varphi}(r, t), p_{r z}(r, t)$, $\sigma_{r r}(r, z, t), \sigma_{z z}(r, z, t), \sigma_{\varphi \varphi}(r, z, t), \sigma_{r z}(r, t)$. To find unknown functions we have to solve system of partial differential equations (19), (20) and (22) with boundary conditions (11).

Taking into account that functions $e_{r r}(r, t), e_{z z}(r, t), e_{\varphi \varphi}(r, t), e_{r z}(r, t)$ are independent of axial coordinate, the derivative $\frac{\partial \sigma_{z z}}{\partial z}$ in the second equilibrium equation has the form:

$$
\frac{\partial \sigma_{z z}}{\partial z}=-\frac{\partial P_{1}(r, z, t)}{\partial z}
$$

Medium motion in the tube is due to pressure gradient

$$
\frac{\partial P_{1}(r, z, t)}{\partial z}=-\psi(t), \psi(0)=0 .
$$

Upon intergrating the above relation, we obtain

$$
P_{1}(r, z, t)=-\psi(t) z+g(r, t) .
$$

Since stress $\sigma_{r z}$ is independent of axial coordinate, the second equilibrium equation can be written as

$$
\frac{\partial \sigma_{r z}}{\partial r}+\frac{\sigma_{r z}}{r}=-\psi(t) .
$$


General solution of this equation has the form

$$
\sigma_{r z}(r, t)=-\frac{\psi(t)}{2} r+\frac{c_{1}(t)}{r}
$$

Stress $\sigma_{r z}$ must be finite at $r=0$, so $c_{1}(t)=0$ and then we have

$$
\sigma_{r z}(r, t)=-\frac{\psi(t)}{2} r
$$

Taking into account (17), we obtain

$$
e_{r z}(r, t)=-\frac{\psi(t)}{4 \mu} r
$$

Further solution of system $(19,20)$ is carried out with the use of finite difference method. Taking into consideration (17), the first equilibrium equation has the form

$$
\frac{\partial g}{\partial r}+2 b\left(\frac{\partial e_{r r}}{\partial r}+\frac{\partial e_{z z}}{\partial r}+\frac{\partial e_{\varphi \varphi}}{\partial r}\right)+2 \mu \frac{\partial e_{r r}}{\partial r}+2 \mu e_{r z} \frac{\partial e_{r z}}{\partial r}+\frac{\mu}{r}\left(2 e_{r r}-2 e_{\varphi \varphi}+3 e_{r z}\right)=0
$$

To find function $g(r, t)$ we need boundary condition

$$
\left.g(r, t)\right|_{r=R}=g_{0}(t),
$$

where $g_{0}(t)$ is the given function of control pressure at the wall of the tube $(r=R)$ in the cross-section $z=0$. We assume that $g_{0}(t)=0$.

Finally we have system of equations $(19,20,30)$ with boundary conditions $(11,31)$. The unknown functions are $u, e_{r r}, e_{z z}, e_{\varphi \varphi}, p_{r r}, p_{z z}, p_{\varphi \varphi}, p_{r z}, g$. To construct finite difference approximation of the obtained system of equations we use central difference for the space derivative and explicit scheme in time. Dimensionless coordinates are used in computations:

$$
\tilde{r}=\frac{r}{R}, \quad \tau=\frac{t}{R} \sqrt{\frac{\mu}{\rho_{0}}}, \quad \tilde{\sigma}_{i j}=\frac{\sigma_{i j}}{\mu},
$$

where $\rho$ is the medium density.

Material parameters have the following values:

$$
\frac{b}{\mu}=4, \quad n=3, \quad \frac{B n R \mu^{n-1} \sqrt{\rho_{0}}}{\sqrt{\mu}}=3.5 .
$$

\section{Results}

The graph of pressure gradient $\psi(\tau)$ is shown in Fig. 1. We assume that deformation process consists of three stages. Firstly pressure gradient increases monotonicaly to the point in time $\tau_{1}$. Then it stays constant to the point in time $\tau_{2}$. After that it decreases monotonicaly and at the point in time $\tau_{3}$ it becomes zero. The points in time have the following dimensionless values: $\tau_{1}=2, \tau_{2}=6, \tau_{3}=8$.

Distributions of reversible deformations $e_{r r}, e_{z z}$ and irreversible deformations $p_{r r}, p_{z z}$ at time points $\tau_{1}, \tau_{2}, \tau_{3}$ are shown in Figs. 2 and 3. Time distributions of reversible deformations $e_{r r}, e_{z z}$ and irreversible deformations $p_{r r}, p_{z z}$ at $r=R$ are presented in Figs. 4 and 5 . Distribution of stresses at the points in time $\tau_{1}, \tau_{2}, \tau_{3}$ is shown in Fig. 6. Components of deformations $e_{\phi \phi}, p_{\phi \phi}$ are close to zero so they are not presented in figures. 


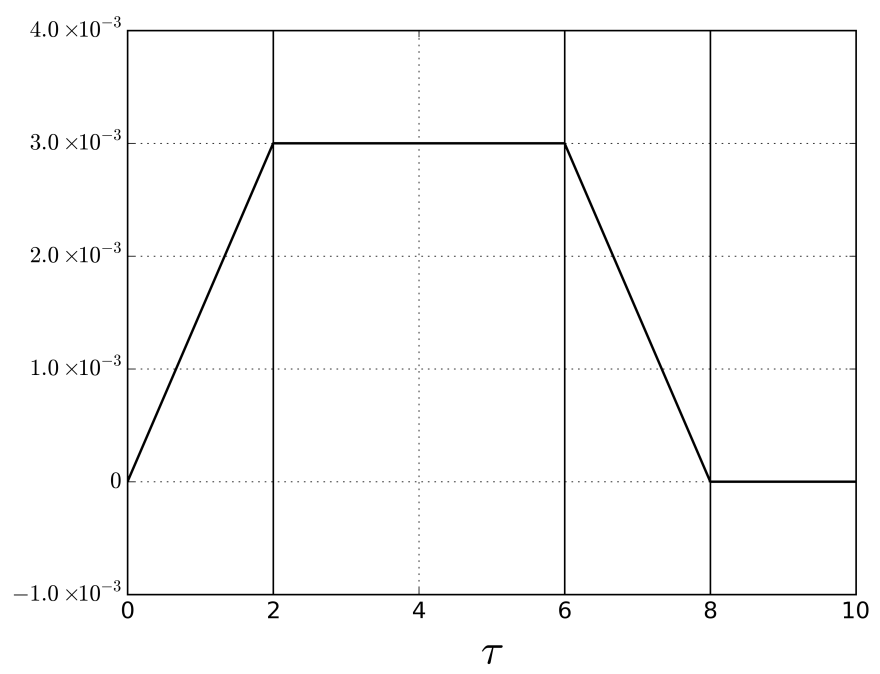

Fig. 1. Pressure gradient

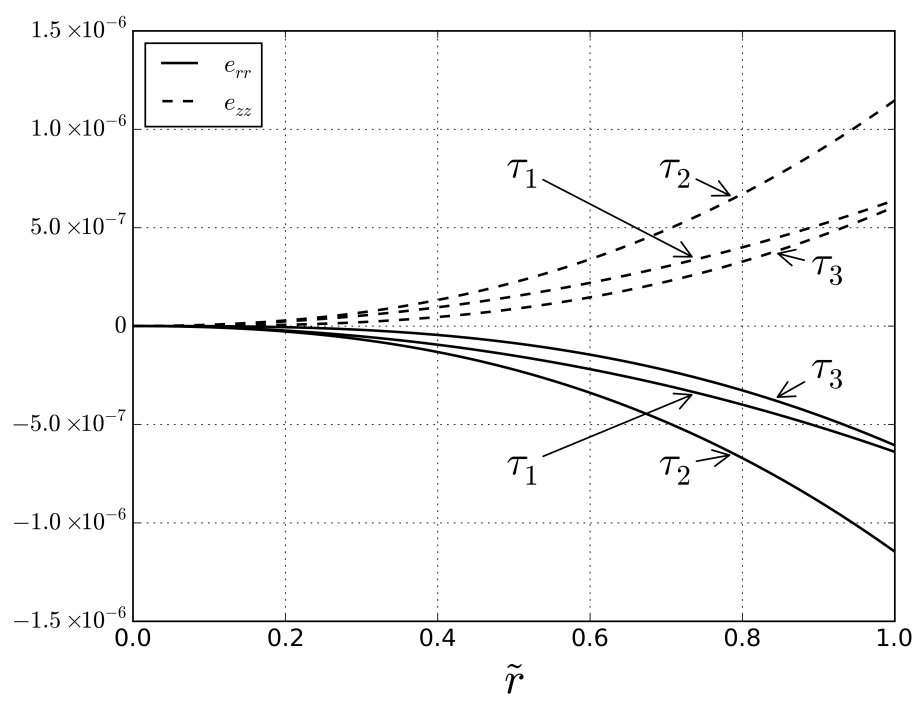

Fig. 2. Distribution of reversible deformations

Reversible and irreversible deformations have roughly the same order of magnitude for chosen duration of the process. It is clear that components of reversible deformations $e_{r r}$ and $e_{z z}$ have almost equal magnitude and differ only in sign. As can be seen from given graphs the first and second stages of the process are characterized by accumulation of reversible and irreversible deformations. An elastic medium springback is clearly noticeable on third stage.

It should be noted that the medium remains in the reversible deformation state even after pressure gradient becomes equal to zero $\left(\tau \geqslant \tau_{3}\right)$. 


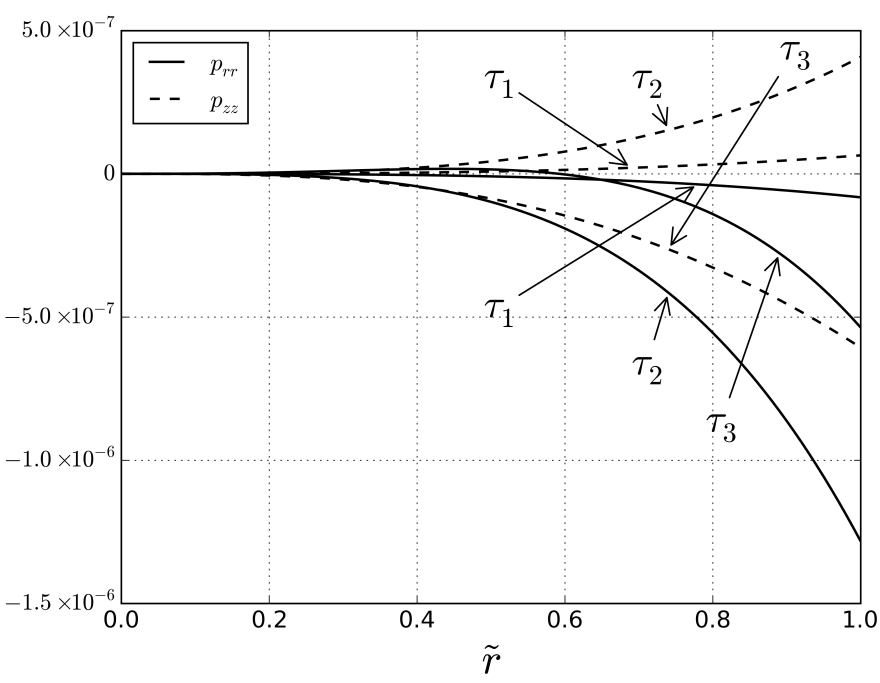

Fig. 3. Distribution of irreversible deformations

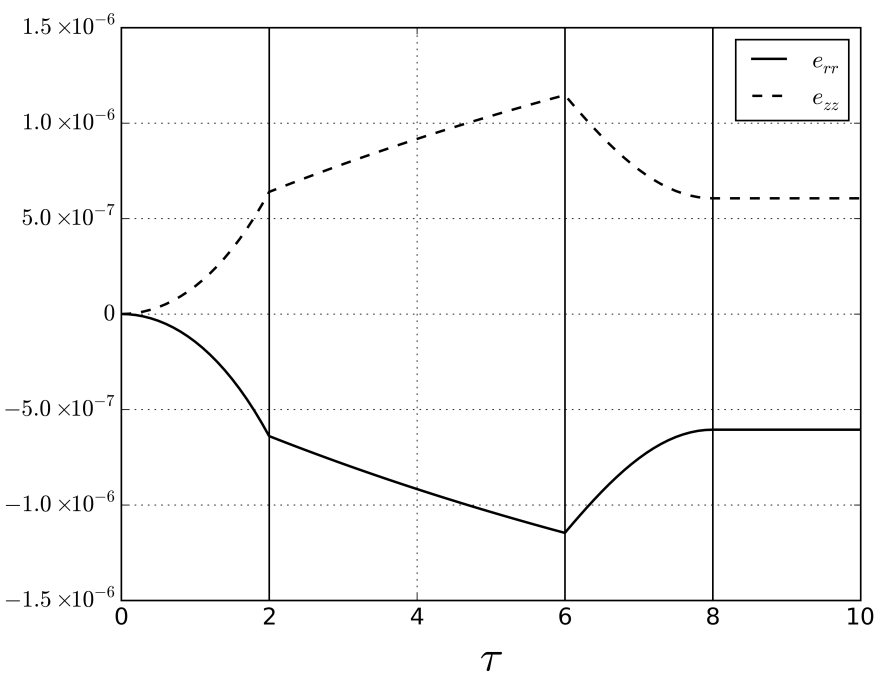

Fig. 4. Reversible deformations at $r=R$

Additionally a series of computations for modified problem are performed. In modified problem we take into account also second order terms in diagonal components of reversible deformation tensor and third and forth order terms in non-diagonal components of reversible deformations tensor. The results of computations show that this refinement of the model has no considerable effect on qualitative characteristics of distribution of stresses and deformations in the medium. Numerical values differ by no more than $10 \%$ from numerical values obtained in solving the original problem. 


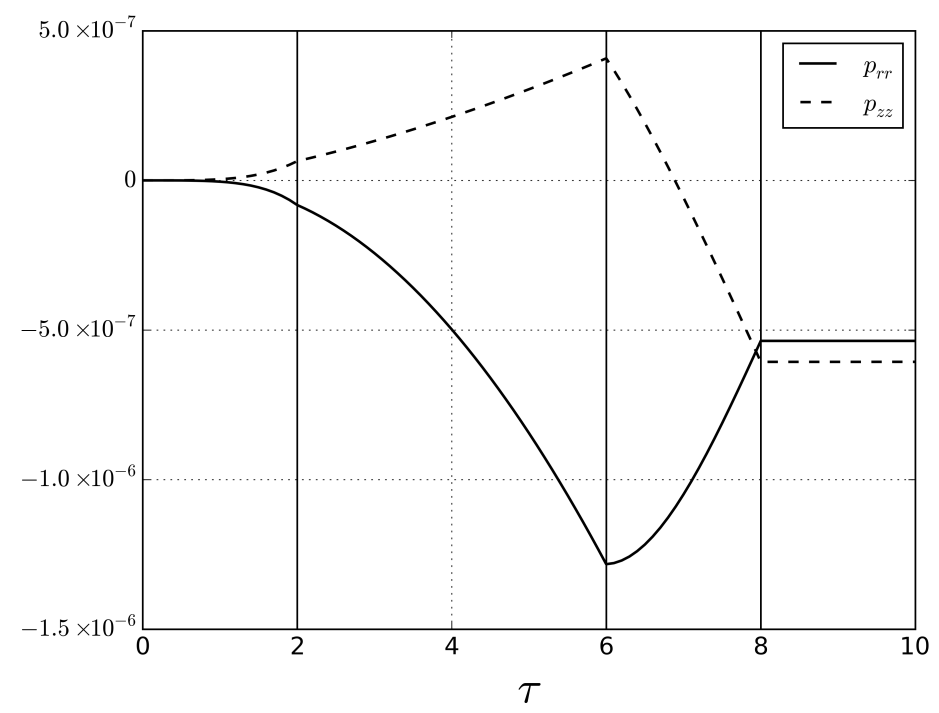

Fig. 5. Irreversible deformations at $r=R$

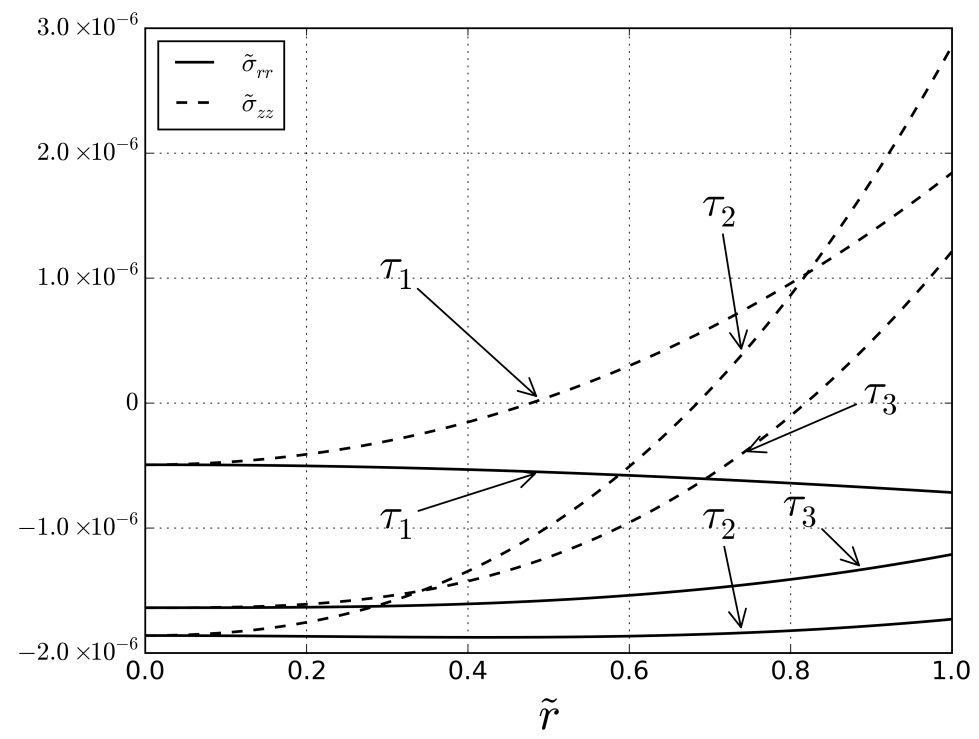

Fig. 6. Distribution of normal stresses

The obtained results can be applied to investigation of drawing processes in manufacturing. In future study we plan to extend the presented mathematical model and take into account plastic flow. 


\section{References}

[1] Yu.N.Rabotnov, Deformable solid mechanics, Moscow, Nauka, 1988 (in Russian).

[2] G.P.Cherepanov, An elastic-plastic problem under conditions of antiplanar deformation, Journal of Applied Mathematics and Mechanics, 26(1962), no. 4, 1040-1057.

[3] B.D.Annin, V.D.Bondar, Antiplane strain in a nonlinearly elastic incompressible body, Journal of Applied Mechanics and Technical Physics, 47(2006), no. 6, 849-856.

[4] V.D.Bondar, Antiplane strain of a body undergoing large-rotations, Journal of Applied Mechanics and Technical Physics, 48(2007), no. 3, 460-466.

[5] V.D.Bondar, Elastoplastic antiplane strain in an incompressible body, Journal of Applied Mechanics and Technical Physics, 55(2014), no. 1, 19-29.

[6] A.A.Burenin, L.V.Kovtanyuk, A.L.Mazelis, Development of a rectilinear axisymmetric viscoplastic flow and elastic aftereffect after its stop, Journal of Applied Mechanics and Technical Physics, 51(2010), no. 2, 261-268.

[7] A.A.Burenin, L.V.Kovtanyuk, The development and deceleration of the flow of an elastoplastic medium in a cylindrical tube, Journal of Applied Mathematics and Mechanics, 77(2013), no. 5, 566-572.

[8] A.A.Burenin, G.I.Bykovtsev, L.V.Kovtanyuk, A simple model of finite strain in an elastoplastic medium, Doklady Physics, 41(1996), no. 3, 127-129.

[9] A.A.Burenin, L.V.Kovtanyuk, Large irreversible strains end elastic aftereffect, Vladivostok, Dalnauka, 2013 (in Russian).

[10] F.N.Norton, The Creep of Steel at High Temperature, McGraw-Hill, New York, 1929.

[11] A.I.Lurie, Theory of Elasticity, Springer, Berlin, 2005.

\section{Антиплоская осесимметричная деформация несжимаемого тела в условиях ползучести}

\section{Сергей Н. Фирсов Александр Н. Прокудин}

\footnotetext{
Рассматривается течение несжимаемой среды в иилиндрической трубе под действием изменяющегося перепада давления. Материал проявляет нелинейные упругие и вязкие свойства. Математическая модель строится с использованием теории больших деформачий, основанной на дифференциальных уравнениях переноса для обратимых и необратимых деформаций. Решение ищется с помощвю аналитических и численных методов.
}

Ключевые слова: большие деформачии, упругость, ползучесть, упругое последействие. 